\title{
Tracheostomy healing time after decannulation
}

\author{
Karen Juelsgaard Christiansen, ${ }^{1}$ Louise Devantier, ${ }^{2,3}$ Thomas Pasgaard, ${ }^{4}$ Thea Emily Benson, ${ }^{5}$ \\ Johanne Juel Petersen, ${ }^{2}$ Thomas Kjargaard, ${ }^{6}$ Michael Pedersen ${ }^{2}$ \\ ${ }^{1}$ Anesthesiology and Intensive Care, Aarhus University Hospital, Aarhus \\ ${ }^{2}$ Comparative Medicine Lab, Department of Clinical Medicine, Aarhus University, Aarhus \\ ${ }^{3}$ Department of Otorhinolaryngology, Region Hospital West Jutland, Holstebro \\ ${ }^{4}$ Intensive Care, Aarhus University Hospital, Aarhus \\ ${ }^{5}$ Department of Public Health, Aarhus University, Aarhus \\ ${ }^{6}$ Otorhinolaryngology, Head and Neck Surgery, Aarhus University Hospital, Aarhus, Denmark
}

Name and location of the institution where the study was performed: Aarhus University Hospital, Aarhus, Denmark.

Background: Prolonged healing of tracheostomy after decannulation has a negative impact on respiration, hygiene, cosmetics, and social life. Even so, evidence-based observations of tracheostoma healing time are lacking. Therefore, the aim of this study was to determine tracheostomy wound healing time after decannulation.

Methods: In this prospective observational cohort study, we included 30 subjects undergoing decannulation following prolonged mechanical ventilation via tracheostomy. Our primary endpoint was tracheostomy healing time defined as time from decannulation to airtight healing. To identify any factors related to healing time, we included information about patient demographics, comorbidities, tracheostomy method, tube size, and intubation time. All subjects were observed daily until their tracheostomy wound had healed.

Results: The median tracheostomy healing time was 6.5 (1-22) days. The duration of tracheal cannulation was the only factor significantly correlated with prolonged healing $(\mathrm{p}=0.03)$. Four patients were subjected to recannulation shortly after decannulation due to hypercapnia, respiratory failure, secretion accumulation, or self-decannulation. All wounds achieved complete spontaneous airtight closure.

Conclusions: Duration of spontaneous tracheostomy closure after decannulation was 1-22 days, and closure time correlated with duration of cannulation.

Key words: Tracheostomy; prolonged mechanical ventilation; decannulation; complication; healing, wound closure.

Correspondence: Karen Juelsgaard Christiansen, Anesthesiology and Intensive Care, Aarhus University Hospital, Palle JuulJensens Boulevard 99, 8200 Aarhus N, Denmark. E-mail: Karen@clin.au.dk

Contributions: KJC, literature search, data collection, manuscript preparation; LD, literature search, study design, manuscript preparation; TP, study design, data collection; TEB, JJP, data analysis, manuscript preparation; TK, formulation of protocol, manuscript preparation; MP, study design, manuscript review. All the authors have read and approved the final version of the manuscript and agreed to be accountable for all aspects of the work.

Conflict of interest: Karen Juelsgaard Christiansen is the co-owner of IntraCair - a spinout company from Aarhus University, Denmark. The other authors report no conflict of interest.

Ethics approval and consent to participate: The Aarhus University Hospital Ethical Committee categorized the study as a quality assurance project (case no: 1-16-02-8-16) and no formal permission to conduct the study was therefore required. Each patient was informed orally about the project and henceforth enrolled with oral consent after decannulation.

Funding: This work was supported by an exploratory pre-seed grant from the Novo Nordisk Foundation. 


\section{Introduction}

Tracheostomy tube decannulation is a significant and critical step in patient recovery after prolonged mechanical ventilation. Timing of decannulation relies on individual clinical evaluation, and no specific guidelines have been published [1-6]. Assessing decannulation readiness may be complicated causing premature decannulation in some patients and resulting in recannulation or oral intubation. After successful decannulation, the tracheostomy is covered with gauze or an occlusive bandage and left to heal spontaneously. Clinical issues during the healing period include impaired phonation and difficulties controlling airway secretion, including a risk of airway infections [7]. The patient is instructed to apply manual pressure to the bandage during cough and speech in order to reinforce cough and phonation. However, manual counter pressure is not always possible for patients suffering from impaired consciousness or limited dexterity [8], and cough strength may have become diminished due to progressive thoracic and abdominal muscular atrophy and muscle fatigue induced by prolonged mechanical ventilation. Furthermore, patients recovering from prolonged mechanical ventilation have shown inability to speak for weeks, leading to a sense of isolation, frustration, anxiety and depression $[2,9]$.

Many patients require frequent wound care due to bandage blow off or accumulation of pulmonary secretions around the stoma site [10]. Hygiene challenges associated with airway secretion may increase the risk of airway infection and respiratory failure, and may therefore heighten the risk of relapse to ventilator therapy $[8,11,12]$. Simple suturing of the skin has not proven effective and even entails a risk of subcutaneous emphysema. Surgical closure of the trachea and suturing of the pre-tracheal tissue are far from the standard of care in the intensive care unit (ICU) [13], albeit some specialists have documented success using a surgical approach for treatment of tracheostomal fistula [14]. A lack of knowledge exists concerning tracheostomy healing time after decannulation. Causal factors affecting the duration of tracheostomal healing remain speculative, and it remains unclear whether a prolonged healing time is associated with an increased risk of pneumonia and with decannulation failure. Thus, the aim of this study was to determine tracheostomy wound healing time after decannulation.

\section{Methods}

This observational prospective cohort study was conducted from August 2016 to August 2019 at Aarhus University Hospital, Denmark.

Inclusion criteria: subjects decannulated in the ICU and subsequently transferred to one of the following departments at Aarhus University Hospital: Department of Cardiology, Department of Cardiothoracic Surgery, Department of Infectious Diseases, or Department of Renal Medicine. Patients were excluded if they were transferred to any other hospital before their tracheostomy wound closure. The local Ethical Committee categorized the study as a quality assurance project (case no: 1-16-02-816) and no formal permission to conduct the study was therefore required.

A total of 30 adult subjects above 18 years of age were included. Two subjects were excluded because they were discharged to local hospitals before airtight healing of their tracheostomy, leaving 28 subjects for analysis in this study. In all cases, the indication for tracheostomy was a need for prolonged mechanical ventilation.
The percutaneous dilation technique was used in 26 patients, whereas an open surgical method was used in the remaining four patients. The decannulation criteria were capping of the tracheostomy tube for a minimum of 24 hours; and subjective assessment of airway secretion, cough effectiveness and ability to swallow properly. The following factors were included in our analyses in order to estimate possible associations with tracheostoma healing time: age, gender, tracheotomy method, short-term bleeding after the tracheotomy procedure, tracheostomy tube size, cannulation time, body mass index, smoking status, immunosuppressive medication, and comorbidity (ischemic heart disease, chronic obstructive pulmonary disease (COPD) and diabetes). The primary outcome measure was time from decannulation to registration of an airtight tracheostomy closure.

\section{Post-decannulation observation of the tracheostomy}

The investigator attended all patients daily for wound inspection and wound rinsing with sterile saline on gauze before application of a new dressing (Aquacel Foam $8 \times 8 \mathrm{~cm}^{2}$, ConvaTec, Flintshire, UK). Patients were encouraged to cough for evaluation

Table 1. Patient characteristics $(n=28)$. Data obtained by inspection of ICU patient notes. An overview of factors analyzed for statistical significance in wound healing time.

\begin{tabular}{|c|c|}
\hline Healing time (days), median [range] & $6.5[1-22]$ \\
\hline Tracheostomy tube treatment (days), median [range] & $15.5[4-93]$ \\
\hline $\begin{array}{c}\text { Sex, n (\%) } \\
\text { Female } \\
\text { Male }\end{array}$ & $\begin{array}{c}4(14) \\
24(86)\end{array}$ \\
\hline Age, median [range] & $60.5[40-89]$ \\
\hline $\begin{array}{l}\text { Smoking status, n (\%) } \\
\text { Never } \\
\text { Former } \\
\text { Current }\end{array}$ & $\begin{array}{l}13(46) \\
12(43) \\
3(11)\end{array}$ \\
\hline $\begin{array}{l}\text { BMI }\left(\mathrm{kg} / \mathrm{m}^{2}\right), \mathrm{n}(\%) \\
\quad<25 \\
\quad 25-30 \\
\quad>30\end{array}$ & $\begin{array}{l}9(32) \\
12(43) \\
7(25)\end{array}$ \\
\hline $\begin{array}{l}\text { Initial tube size, } \mathrm{n}(\%) \\
\quad 7 \mathrm{~mm} \\
8 \mathrm{~mm}\end{array}$ & $\begin{array}{c}2(7) \\
26(93)\end{array}$ \\
\hline $\begin{array}{l}\text { Downsized tube, n (\%) } \\
\text { No } \\
\text { Yes }\end{array}$ & $\begin{array}{l}11(39) \\
17(61)\end{array}$ \\
\hline $\begin{array}{l}\text { Diabetes, n (\%) } \\
\text { No } \\
\text { Yes }\end{array}$ & $\begin{array}{c}20(71) \\
8(29)\end{array}$ \\
\hline $\begin{array}{l}\text { COPD, n (\%) } \\
\text { No } \\
\text { Yes }\end{array}$ & $\begin{array}{c}23(82) \\
5(18)\end{array}$ \\
\hline $\begin{array}{l}\text { Ischemic heart disease, n (\%) } \\
\text { No } \\
\text { Yes }\end{array}$ & $\begin{array}{l}28(64) \\
10(36)\end{array}$ \\
\hline $\begin{array}{l}\text { In-hospital immunosuppressants, n (\%) } \\
\text { No } \\
\text { Yes }\end{array}$ & $\begin{array}{c}20(71) \\
8(29)\end{array}$ \\
\hline $\begin{array}{l}\text { Bleeding complications, } \mathrm{n}(\%) \\
\text { No } \\
\text { Yes }\end{array}$ & $\begin{array}{c}23(82) \\
5(18)\end{array}$ \\
\hline
\end{tabular}

BMI, body mass index; COPD, chronic obstructive pulmonary disease. 
of audible air leakage from the stoma site. In case of doubt, a sterile transparent adhesive film (Tegaderm Film, $6 \times 7 \mathrm{~cm}^{2}, 3 \mathrm{M}$, Neuss, Germany) was applied, and the patient was instructed to perform the Valsalva maneuver. All tests were conducted by a single investigator (KJC) to eliminate any investigator bias. Ward staff was instructed to maintain usual tracheostomy care.

Wound closure was assessed by inspection of air accumulation under the film. If the patient had signs of sore skin or pain related to removal of the dressing, a skin barrier film (Cavilon, 3M) was applied. A few patients were unable to cooperate performing the Valsalva maneuver or were unable to cough, in which cases airtight closure was determined by lack of visible or audible air bubbles when applying the film. The ward staff was informed about study completion on the day of airtight healing, and any dressing preferred by the ward staff could be used, as needed.

\section{Statistical analysis}

Continuous data not following a normal distribution are presented as median and range. Q-Q plots were used to check continuous values for normality. Categorical data are presented as frequencies and corresponding percentages. To evaluate potential predictors related to tracheostoma healing time, unpaired Student's $t$ test was used for dichotomous variables, and one-way analysis of variance was used for categorical variables. A simple linear regression model was applied for continuous variables along with an estimation of the Pearson's correlation coefficient. Non-parametric data were log-transformed. A $\mathrm{p}<0.05$ was considered statistically significant. We used a statistical software package (STATA/IC 14.1, StataCorp. LP, College Station, TX, USA).

\section{Results}

Duration of cannulation varied between four and 93 days (median 15.5 days) and was the only factor correlating with prolonged tracheostomy healing $(\mathrm{p}=0.03)$ (Table 2). Table 3 shows primary diagnosis and department affiliation at admission to hospital, along with healing days. No correlation between specialty or primary diagnosis and healing time reached statistical significance. Four patients were subjected to recannulation and readmission to mechanical ventilation shortly after decannulation, and one patient was also orally intubated. These courses are illustrated in Figure 1.

Decannulation periods before recannulation varied from zero

Table 2. Correlation coefficients and statistical analysis of potential predictors and healing time of tracheostoma wound among study participants $(\mathbf{n}=\mathbf{2 8})$.

\begin{tabular}{lcc} 
Determinants of healing time & $\boldsymbol{R}$ & $\mathbf{p}$ \\
Tracheostomy treatment (days) & 0.41 & 0.03 \\
Sex & - & 0.75 \\
\hline Age (years) & -0.24 & 0.23 \\
Smoking status & - & 0.90 \\
\hline BMI $\left(\mathrm{kg} / \mathrm{m}^{2}\right)$ & 0.08 & 0.68 \\
Downsized tube & - & 0.69 \\
\hline Diabetes & - & 0.85 \\
Chronic obstructive lung disease & - & 0.13 \\
\hline Ischemic heart disease & - & 0.75 \\
In-hospital immunosuppressants & - & 0.69 \\
\hline Bleeding complications & - & 0.62 \\
\hline
\end{tabular}

to six days. The main reasons for recannulation of our study subjects were hypercapnia, respiratory failure, secretion accumulation and patient self-decannulation. Patients no. 1 and no. 2 were recannulated once, and patient no. 3 was recannulated twice. Patient no. 4 was recannulated in the stoma thrice and orally intubated once due to accumulation of secretion and tracheostomal air leakage. Therefore, the fourth period of mechanical ventilation was performed by translaryngeal intubation ten days after decannulation with cuff placement below the level of tracheostomy to optimize tracheostomy wound healing. The tracheostomy healing time for this patient was 16 days.

\section{Discussion}

This was a prospective observational cohort study. Interobserver bias was eliminated by using a single investigator. Our study failed to disclose any factors that may accelerate tracheostomy healing, but in lieu of prolonged and repeated intubations after decannulation, fast tracheostomy closure may be a critical factor in achieving a successful post-decannulation course. Several clinical studies have investigated the impact of tracheotomy technique, early versus late tracheotomy, and criteria for the timing of decannulation $[8,15-18]$; however, the intuitively critical phase from decannulation to the tracheostomy has healed completely and is surprisingly under-investigated. This limited prospective observational study indicated that a need exists for larger interventional studies focusing on reducing tracheostomy healing time.

\section{Decannulation criteria}

Currently, the evaluation of the tracheostomy patients' readiness for decannulation is largely based on factors, including airway secretion, cough effectiveness, ability to swallow, ability to tolerate tube capping, etiology of respiratory failure, difficulty of intubation, oxygen requirements, respiratory rate, comorbidities and age $[3,17,19-21]$. Striving to take all these factors into account may prolong cannulation time. In our study, we found a positive correlation between cannulation time and prolonged tracheostomy wound healing time, indicating that early decannulation may advance patient recovery. Our list of decannulation criteria included only four factors: capped tracheostomy tube for $24 \mathrm{~h}$, low secretion volume, effective cough, and ability to swallow.

While testing for tube capping continuously for $24 \mathrm{~h}$, we obser-

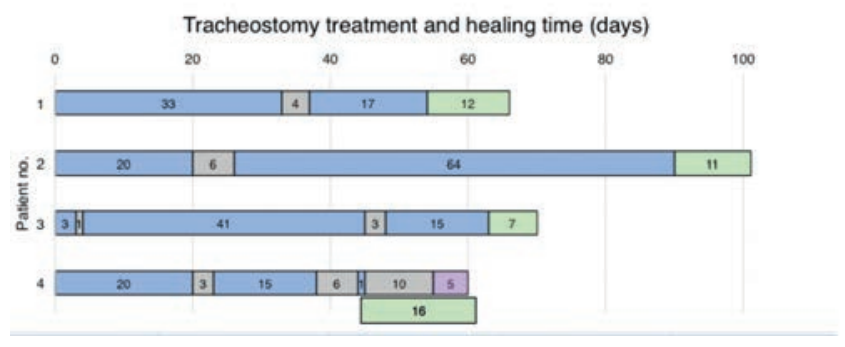

Blue line, tracheostomy treatment; grey area, decannulation; green area, time from decannulation to complete stomal closure; purple area, oral intubation; 1 day, intubated and decannulated (vice versa) on the same day. Patient no. 4 was orally intubated for five days (purple); and his time from decannulation to complete stomal closure was 16 days (green).

Figure 1. Patients with more than one course of tracheostomy tube treatment. Data produced by study investigator through daily patient observation. An overview of patient recannulation and/or oral intubation courses. 
ved several clinical parameters, including $\mathrm{PaO}_{2}(>70 \mathrm{mmHg})$, normocapnia (hypercapnia was accepted in patients who habitually presented with high levels of carbon dioxide), and arterial blood $\mathrm{pH}$ (in the 7.32-7.45 range). Furthermore, we assessed hemodynamics, infection status, and level of consciousness. We evaluated airway secretion primarily by volume, but also by color, viscosity, odor, and microbiological analysis. Coughing was assessed both auditorily and indirectly by the frequency of need for tracheostomal suction. If patients had no need for suctioning for eight hours, they were considered capable of clearing their airways on their own. Finally, swallowing was evaluated by observing for absence of dysphagia.

Approaches in tracheostomy weaning are determined not only by local procedures but also by how hospitals are organized, e.g., some hospitals have dedicated teams for tracheostomy follow up, others have step-down units, or provide long-term care for tracheostomy patients. This variation in clinical procedures and organization of tracheostomy care complicates comparative studies aiming to obtain evidence-based knowledge about early successful decannulation.

\section{Cannulation time related to healing time}

Tracheostomy tube treatment time (cannulation time) was related to tracheostomy closure time, supporting the view by Bishop et al. [22] that patients who have a tracheostomy tube for a prolonged period of time are more susceptible to developing a persistent stoma than are other patients. Occurrence of slow healing may, however, generally reflect a poor healing performance, which may be influenced by factors such as comorbidities, smoking status, BMI, etc. Thus, a long tube time may potentially indicate other factors actually predictive of a longer healing time.

Though shorter cannulation time was found to be a statistically significant predictor of early wound closure, we suggest that caution is warranted given our small study group size. Other factors were considered: age, gender, tracheotomy method, short-term bleeding after the tracheotomy procedure, tracheostomy tube size, BMI, smoking status, immunosuppressive medication, and comorbidity, but these factors did not correlate with healing time with this relatively small number of included subjects.

\section{Decannulation failure}

Recannulation was not an endpoint in this study. However, we

Table 3. Primary diagnoses and healing time. Data obtained by inspection of ICU patient notes. An overview of patient admission, divided into Medical and Surgical Specialties, Primary Diagnoses, and Tracheostomy Healing Time.Determinants of healing time

\begin{tabular}{|c|c|c|}
\hline Specialty & Primary diagnosis at the time of admission to the ICU & Healing time (days) \\
\hline Cardiology & MI & 16 \\
\hline Cardiology & MI + IMPELLA & 4 \\
\hline Cardiology & MI & 7 \\
\hline Cardiology & MI complicated by VSD & 19 \\
\hline Cardiology & Cardiac arrest & 3 \\
\hline Thoracic surgery & Aorta valve replacement & 4 \\
\hline Thoracic surgery & PTE & 11 \\
\hline Thoracic Surgery & Mitral valve replacement + CABG & 7 \\
\hline Thoracic Surgery & Aortic valve replacement & 2 \\
\hline Thoracic Surgery & Aortic valve replacement + CABG & 9 \\
\hline Thoracic Surgery & Aortic dissection & 16 \\
\hline Thoracic Surgery & Aortic dissection & 17 \\
\hline Thoracic Surgery & Thoracic and abdominal aortic aneurysm & 4 \\
\hline Thoracic Surgery & Trauma sternum + costae fracture & 3 \\
\hline Cardiology/Thoracic Surgery & HTX & 12 \\
\hline Cardiology/Thoracic Surgery & HTX & 4 \\
\hline Cardiology/Thoracic Surgery & HTX & 22 \\
\hline Cardiology/Thoracic Surgery & Heart failure (LVAD) & 15 \\
\hline Cardiology/Thoracic Surgery & HTX & 7 \\
\hline Esophagus Surgery & Esophagus cancer & 1 \\
\hline Esophagus Surgery & Esophagus cancer & 3 \\
\hline Esophagus Surgery & Esophagus perforation & 6 \\
\hline Pulmonary Medicine & Pneumonia (Wegener's gran.) & 1 \\
\hline Pulmonary Medicine & Pneumonia & 2 \\
\hline Pulmonary Medicine & Respiratory failure (Wegener's gran.) & 10 \\
\hline Pulmonary Medicine & Pneumonia (Wegener's gran.) & 6 \\
\hline Infectious Diseases & Odontoid fracture + paravertebral abscess & 11 \\
\hline Infectious Diseases & Pneumococcal meningitis & 5 \\
\hline
\end{tabular}

MI, myocardial infarction; IMPELLA, implantation of intracardial assist device; VSD, ventricular septal defect; PTE, pulmonary thromboendarterectomy; CABG, coronary artery bypass grafting; HTX, heart transplantation; LVAD, left ventricular assist device. 
observed recannulation in four of 28 subjects, exceeding $2-5 \%$ which is considered an acceptable recannulation rate [20]. A recent study including 50 patients demonstrated a similar rate [11]. The wide variation in failure rates may be due to a lack of agreement on the time-point for assessing failure [23]. A previous study reported failure rates between $3.5 \%$ and $32 \%$ with registration of recannulation within 1-60 days [5]. In our study, we observed that recannulation was performed on day 0-6 after decannulation (Figure 1). From our results, it remains unclear what caused decannulation failure. This finding merely emphasizes just how difficult assessment of decannulation readiness is. Furthermore, it remains unclear which factors are responsible for wound closure time. Therefore, patient characteristics and tracheostomy decannulation assessment criteria should be considered when investigating decannulation failure in a larger cohort study.

\section{Patients' characteristics}

Our study population primarily consisted of patients with complex cardio or cardiothoracic disease. These patients often present with multiorgan failure, requiring treatment at highly specialized hospitals such as the Aarhus University Hospital. The study subjects were included at the time of decannulation. Thus, patients who were eligible for admission to less specialized hospitals before their decannulation were unable to enter our study population. This may have produced a study population comprising patients who were more fragile than the standard tracheostomy patient. However, we did not include neurological or neurosurgical patients who often require long ICU admission times and neurorehabilitation. In neurological tracheostomy patients, we expect higher rates of recannulation, or even complete decannulation failure. This should be acknowledged when concluding on our results or comparing our findings with those of other studies.

\section{Respiratory physiology in open tracheostomy condition}

Generally, tracheostomized patients come from a multitude of clinical specialties, and decannulation often coincides with transferal from the ICU to a general ward. Due to the change in staffing, details in vital parameters and deterioration of respiratory capacity may be challenging to monitor.

It remains unknown to which extent the change in upper airway physiology derived from the open tracheostomy triggers the development of post-decannulation respiratory failure. One prerequisite for an effective cough is an airtight airway without fistulae and effective glottic closure at the onset of the expiratory effort [24]. Accordingly, an insufficiently sealed tracheostomy impairs cough and regulation of positive end-expiratory pressure (PEEP).

The opening into the trachea below the vocal cords is undoubtedly particularly debilitating for this vulnerable group of patients. Tube downsizing before decannulation partly addresses the clinical issue by increasing the luminal space around the tube, ensuring a freer airway while allowing the tracheostoma to narrow its diameter. Intuitively, a method that immediately seals the tracheostomy without accumulating secretion while accelerating tracheostomy healing after decannulation seems an attractive option.

\section{Study limitations}

Due to our relatively low number of study subjects, we cannot calculate either statistical correlation or $\mathrm{p}$ between patient demographics or tracheostomy data and healing time. Drawing on our current results, we can, however, suggest associations that need further investigation. Further larger studies should be performed to produce firm conclusions regarding factors that may influence tracheostomy wound healing time. Our study population predominantly consisted of male subjects (86\%) with cardiac, thoracic, or cardiothoracic primary diagnoses $(68 \%)$. Extrapolation of our results must therefore be considered keeping this in mind.

The need for consensus on decannulation criteria must be mentioned as this directly affects tracheostomy tube time, which was the only significant factor in wound closure time in our results. The fact that decision making concerning decannulation is rooted in expert opinion rather than based on universally accepted objective guidelines emphasizes how delicate this assessment is. Using quantitative or semi-quantitative scoring systems that take into account, e.g., peak cough flow, maximum expiratory pressure, and state of consciousness, might delay decannulation rather than optimize results, as treating doctors need to assess long lists of decannulation criteria. Our results suggest that decannulation should take place as early as possible to ensure early wound closure and thereby accelerated patient recovery.

\section{Conclusion}

Our study demonstrated that the duration of tracheostomy healing after decannulation was related to cannulation time, but a need exists for further investigation of factors related to delayed healing and of measures leading to accelerated healing.

\section{Acknowledgements}

The authors express their gratitude to Professor Jens Christian Djurhuus (Aarhus University) for scientific supervision regarding the study design and for critical review of the manuscript, and to Professor John Michael Hasenkam (Aarhus University) for critical review of the manuscript.

\section{Abbreviations \\ ICU: intensive care unit; COPD: chronic obstructive pulmonary disease; PEEP: Positive end expiratory pressure; BMI: body mass index.}

\section{References}

1. Ceriana P, Carlucci A, Navalesi P, Rampulla C, Delmastro M, Piaggi G, et al. Weaning from tracheotomy in long-term mechanically ventilated patients: feasibility of a decisional flowchart and clinical outcome. Intens Care Med 2003;29:8458.

2. Christopher KL. Tracheostomy decannulation. Respir Care 2005;50:538-41.

3. Stelfox HT, Crimi C, Berra L, Noto A, Schmidt U, Bigatello LM, et al. Determinants of tracheostomy decannulation: an international survey. Crit Care 2008;12:R26.

4. Hess DR, Altobelli NP. Tracheostomy tubes. Respir Care 2014;59:956-71; discussion 71-3.

5. Singh RK, Saran S, Baronia AK. The practice of tracheostomy decannulation-a systematic review. J Intens Care 2017;5:38.

6. Marchese S, Corrado A, Scala R, Corrao S, Ambrosino N. Tracheostomy in patients with long-term mechanical ventilation: a survey. Respir Med 2010;104:749-53.

7. Caronia FP, Fiorelli A, Santini M, Castorina S. A persistent tra- 
cheocutaneous fistula closed with two hinged skin flaps and rib cartilage interpositional grafting. Gen Thorac Cardiovasc Surg 2016;64:625-8.

8. Choate K, Barbetti J, Currey J. Tracheostomy decannulation failure rate following critical illness: a prospective descriptive study. Aust Crit Care 2009;22:8-15.

9. Freeman-Sanderson AL, Togher L, Elkins M, Kenny B. Quality of life improves for tracheostomy patients with return of voice: A mixed methods evaluation of the patient experience across the care continuum. Intens Crit Care Nurs 2018;46:106.

10. Freeman S. Care of adult patients with a temporary tracheostomy. Nurs Stand 2011;26:49-56; quiz 8.

11. Bishnoi T, Sahu PK, Arjun AP. Evaluation of factors determining tracheostomy decannulation failure rate in adults: an Indian perspective descriptive study. Indian J Otolaryngol Head Neck Surg 2020:1-6.

12. Heffner JE. Tracheostomy decannulation: marathons and finish lines. Crit Care 2008;12:128.

13. Lopez-Pastorini A, Kraja O, Ludwig C, Plönes T, Storre JH, Rommel T, et al. [Reduction of tracheotomy associated tracheal stenosis by surgical closure of the tracheostomy].[Article in German]. Pneumologie 2015;69:335-40.

14. Eliashar R, Sichel JY, Eliachar I. A new surgical technique for primary closure of long-term tracheostomy. Otolaryngol Head Neck Surgery 2005;132:115-8.

15. Cheung NH, Napolitano LM. Tracheostomy: epidemiology, indications, timing, technique, and outcomes. Respir Care 2014;59:895-915; discussion 6-9.
16. Cipriano A, Mao ML, Hon HH, Vazquez D, Stawicki SP, Sharpe RP, et al. An overview of complications associated with open and percutaneous tracheostomy procedures. Int J Crit Illn Inj Sci 2015;5:179-88.

17. Hernández Martínez G, Rodriguez ML, Vaquero MC, Ortiz R, Masclans JR, Roca O, et al. High-flow oxygen with capping or suctioning for tracheostomy decannulation. N Engl J Med 2020;383:1009-17.

18. Young D, Harrison DA, Cuthbertson BH, Rowan K. Effect of early vs late tracheostomy placement on survival in patients receiving mechanical ventilation: the TracMan randomized trial. JAMA 2013;309:2121-9.

19. Stelfox HT, Hess DR, Schmidt UH. A North American survey of respiratory therapist and physician tracheostomy decannulation practices. Respir Care 2009;54:1658-64.

20. Heffner JE. The technique of weaning from tracheostomy. Criteria for weaning; practical measures to prevent failure. J Crit Illn 1995;10:729-33.

21. Mitchell RB, Hussey HM, Setzen G, Jacobs IN, Nussenbaum B, Dawson C, et al. Clinical consensus statement: tracheostomy care. Otolaryngol Head Neck Surg 2013;148:6-20.

22. Bishop JB, Bostwick J, Nahai F. Persistent tracheostomy stoma. Am J Surg 1980;140:709-10.

23. Thomas AJ, Talbot E, Drewery H. Failure of standard tracheostomy decannulation criteria to detect suprastomal pathology. Anaesth Rep 2020;8:67-70.

24. McCool FD. Global physiology and pathophysiology of cough: ACCP evidence-based clinical practice guidelines. Chest 2006;129:48S-53S.

Received for publication: 26 November 2021. Accepted for publication: 11 January 2022.

This work is licensed under a Creative Commons Attribution-NonCommercial 4.0 International License (CC BY-NC 4.0).

(C) Copyright: the Author(s), 2022

Licensee PAGEPress, Italy

Multidisciplinary Respiratory Medicine 2022; 17:822

doi:10.4081/mrm.2022.822 